\title{
Acute and Cumulative Effects of Different Times OF ReCOVERY From Whole Body VibRation Exposure on Muscle Performance
}

\author{
Marzo E. Da Silva-Grigoletto, ${ }^{1}$ Diana M. Vaamonde, ${ }^{2}$ Eduardo Castillo, ${ }^{2}$ \\ Maria S. Poblador, ${ }^{2}$ Juan M. García-Manso, ${ }^{3}$ and Jose L. Lancho ${ }^{2}$ \\ ${ }^{1}$ Andalusian Center of Sports Medicine, Córdoba, Spain; ${ }^{2}$ Morphofunctional Sciences in Sports Laboratory, Morphological \\ Sciences Department, School of Medicine, Universidad de Córdoba, Córdoba, Spain; and ${ }^{3}$ Physical Education Department, \\ School of Physical Activity and Sport Sciences, Universidad de Las Palmas de Gran Canaria, Gran Canaria, Spain
}

\begin{abstract}
Da Silva-Grigoletto, ME, Vaamonde, DM, Castillo, E, Poblador, MS, García-Manso, JM, and Lancho, JL. Acute and cumulative effects of different times of recovery from whole body vibration exposure on muscle performance. J Strength Cond Res 23(7): 2073-2082, 2009-This experiment was designed to assess the acute (Study I) and cumulative response (Study II) of muscle performance to differing recovery times after exposure to whole body vibration (WBV). All subjects (mean age $19.7 \pm 1.9$ ) were healthy and physically active. In both studies, subjects were exposed to a WBV bout of 6 exposures of 60 seconds each, with frequency of $30 \mathrm{~Hz}$ and amplitude of $4 \mathrm{~mm}$. In Study I, subjects $(n=30)$ underwent 3 trials ( 1 per day) on different days with a 2-day wash-out period between trials; each trial included either a 1, 2, or 3 minutes of recovery between exposures to WBV. All subjects underwent all trials, which were randomly assigned. Jump ability and muscle power were measured before and after each bout. In Study II, subjects $(n=45)$ underwent 12 sessions of WBV training in 4 weeks ( 3 bouts/ $w k$ ). The subjects were randomly assigned to 1 of the following 3 groups: WBV with 1-minute recovery periods between exposures, WBV with 2-minute recovery periods between exposures, or control group. Jump ability, muscle power, and strength were measured before and after each bout. In the acute study (I), recovery times of 1 and 2 minutes enhanced all measured parameters $(p<0.05)$, with the 2 -minute recovery being more effective. In the long-term study (II), however, although both periods also enhanced the measured parameters $(p<0.05)$, the 1 -minute recovery proved more effective because the response was modified by systematic stimulation.
\end{abstract}

Address correspondence to Marzo Edir Da Silva-Grigoletto, pit_researcher@yahoo.es.

23(7)/2073-2082

Journal of Strength and Conditioning Research

(C) 2009 National Strength and Conditioning Association
In conclusion, 2-minute recovery periods provided the most effective acute enhancement of muscle activation, whereas the 1-minute recovery provided a more effective cumulative enhancement of muscle power and jump ability.

KEY WoRDS training, muscular strength, muscular power

\section{INTRODUCTION}

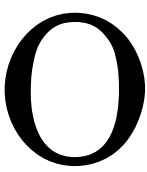

ptimization or modification of the body's functional capacity leads to improved performance (61). Workload-induced stimuli play a major role in the training process. The search for new, specific, and innovative technologies or methods represents a constant challenge to sports technicians and has already yielded interesting findings. In recent years, whole body vibration (WBV) platforms have been incorporated into physical training programs (19) as a means of enhancing muscle strength $(3,4,56,58,20,60)$. Because much contradiction exists as to whether WBV is beneficial $(43,48)$ and as to which parameters may be the most suitable for producing benefits, much current research centers on the development of specific training protocols based on vibration stimulus. Recent studies have addressed, among other things, the optimal frequency of vibration $(12,18)$. However, work still needs to be done on making stimuli more efficient, with a view to enhancing the acute or cumulative response to WBV (28). Likewise, of the variables traditionally used to characterize training loads (volume, intensity, duration, and recovery), optimization of the latter 2 variables poses a considerable challenge to strength and conditioning coaches opting to use WBV.

To the best of our knowledge, no published studies deal specifically with recovery or with its effect on the acute and cumulative strength response to WBV. A review by Luo et al. (36) analyzing various parameters used in this type of training (frequency, amplitude, duration, and vibration method used) makes no mention of recovery as a part of research. It is well 


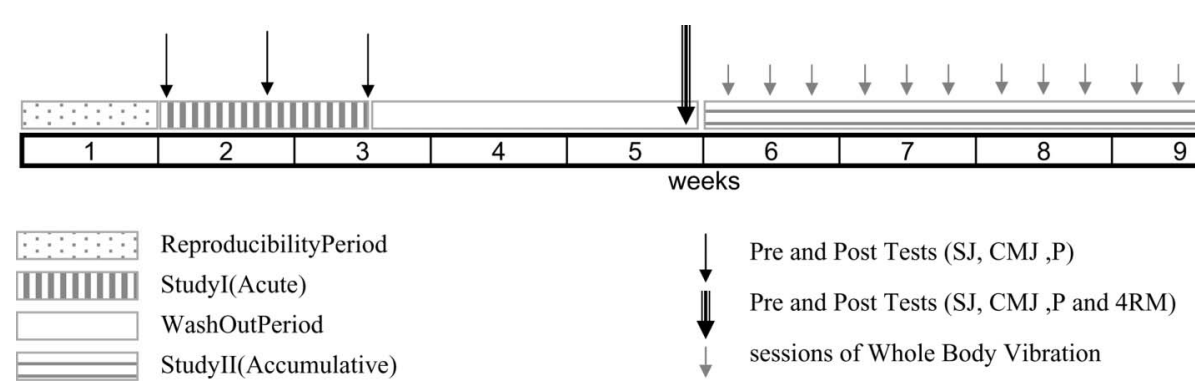

Figure 1. General layout and timing of studies, including training sessions and tests performed: squat jump (SJ), countermovement jump (CMJ), muscular power $(\mathrm{P})$, and 4 repetition maximum $(4 \mathrm{RM})$.

known that physical exercise generates states of fatigue (central or peripheral) whose intensity is governed by the magnitude and duration of the stimulus (51); the application of vibration stimuli can produce a similar fatigue (52). Because fatigue is a common feature of any physical exercise, the sports technician aims to recover initial homeostasis, and even to improve upon initial values, when seeking to optimize training loads. The interval required to achieve a return to initial values is termed the recovery phase $(62,46)$.

As with any other strength training technique, the rest period applied after any WBV exercise is intended to regain the muscle's mechanical potential and its functional ability to develop the tension required. More specifically, the aim is that when repeating a stimulus or applying force during a sporting activity the muscle should be in a state of maximal postactivation potentiation (PAP), thus favoring optimally efficient contraction $(59,26)$. The duration and kinetics of the recovery period in any training program are determined by a number of factors, some endogenous (e.g., age, muscle volume, fiber type) and others exogenous to the athlete (e.g., prior fatigue level, years of training, prior vibration-training experience). Recovery is thus dependent on the principles of individuality and specificity that should guide the design of any training program (57); both principles are involved in determining the acute and cumulative response to training of varying duration and intensity.

This study is based on the hypothesis that the duration of the recovery interval is affected by the number of times that the vibration stimulus is applied during training. With that in mind, the following were examined in young physically active males: (a) the acute response of muscle performance to differing recovery times after WBV; (b) the effect of short-term training on optimal recovery times after WBV.

\section{Methods}

\section{Experimental Approach to the Problem}

The whole experimental procedure lasted 10 weeks. After a first week devoted to reproducibility testing, the first study was performed to evaluate the effect of different rest intervals on acute power response (measured by jumping and squat tests); for this purpose, a repeated measures design was used. After completion of the acute study (Study I), a 3-week washout period was applied to avoid any carryover effect. With use of the results derived from the acute study, a randomized longitudinal study (Study II) was performed to assess the cumulative effect on power and strength (4 repetition maximum $[4 \mathrm{RM}]$ ) production (Figure 1).

\section{Subjects}

Forty-five young male subjects volunteered to participate in the study (30 in Study I and 45 in Study II). The mean $(S D)$ characteristics of the subjects are shown in Table 1.

Medical histories were reviewed by a doctor to assess suitability for the study, and each subject completed a questionnaire on physical activity. Subjects with osteoarticular conditions (including fracture or injury) were excluded. Participants were informed about the study procedure and its possible risks and benefits and signed a consent form approved by the University of Córdoba Ethics Committee. All subjects were physically active and engaged in various sporting activities in university teams (nonprofessional) but had not 


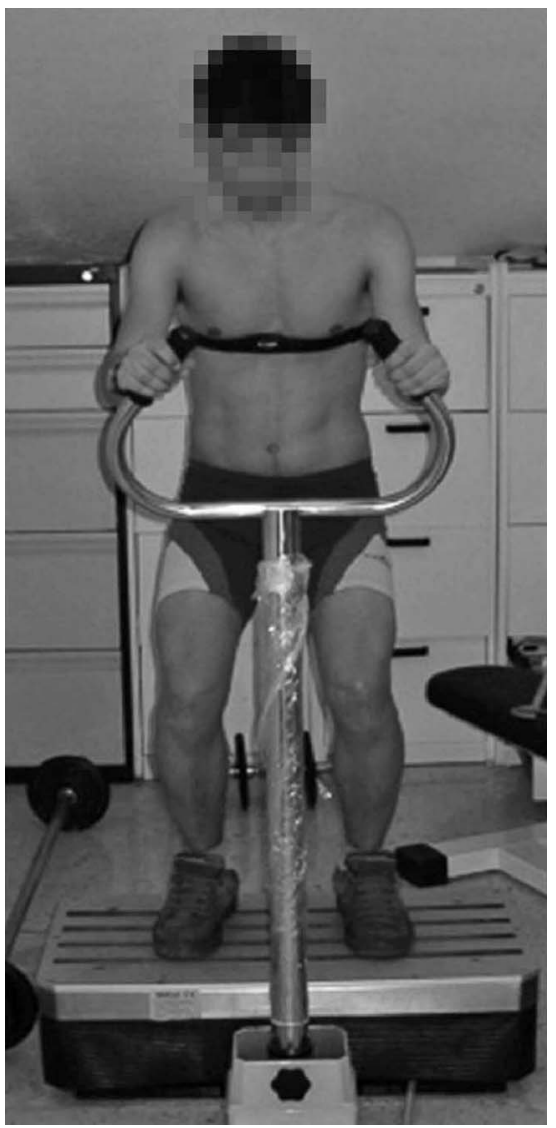

Figure 2. Subject in half squat position on vibration platform.

been engaged in regular resistance or jump-training programs for the last 12 months. All subjects had a very similar training volume (min $3 /$ wk and $\max 4 / \mathrm{wk}$ ), spread into 1-hour sessions. The sports they practiced were the following: indoor soccer $(n=26)$, basketball $(n=12)$, volleyball $(n=3)$, and paddle ball $(n=4)$. None of the selected subjects performed specific physical preparation exercises because, given the category they play in and the small amount of time they train, training was focused on game exercises. All research was undertaken during the in-season period.

\section{Tests Performed}

The first 3 tests described below (jump tests and muscular power test) were performed in Study I and Study II. The last test (4RM) was performed only in Study II.

All tests were preceded by a 5 -minute warm-up $(3 \mathrm{~min}$ $25 \mathrm{~W}+2 \min 50 \mathrm{~W}$ ) on a cycloergometer (Ergoline 900, Ergometrics, Bitz, Germany) followed by a 5-minute program of stretching for femoral quadriceps, hamstrings, and triceps surae.

Jump Tests. Lower-body explosive strength characteristics, expressed as elevation of the body's center of gravity (vertical

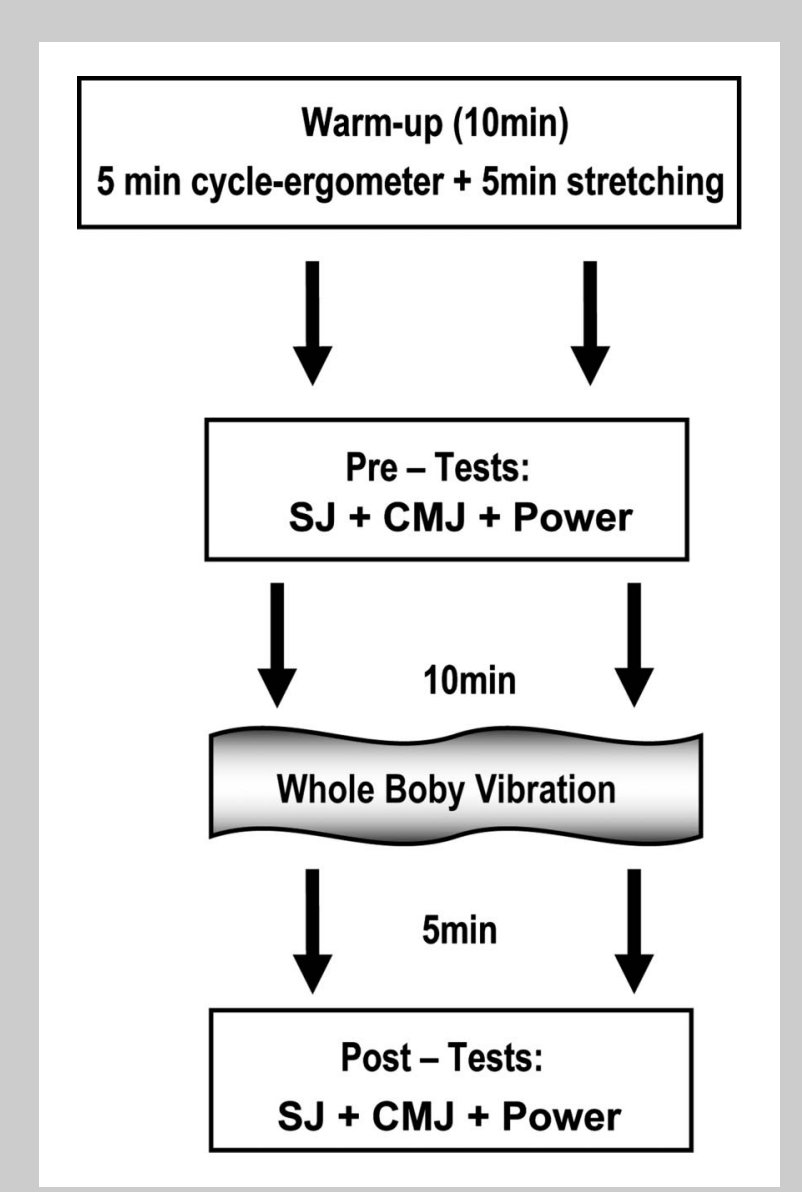

Figure 3. Data-recording protocol for Study I.

jump), were assessed using an infrared-ray platform (A.F.R technology) built into the MuscleLab system (Model PFMA 3010e, Ergotest, Langesund, Norway).

Two different vertical jumps were used for data recording: squat jump (SJ) and countermovement jump (CMJ) (7,31). The $\mathrm{SJ}$ is a test used to assess lower-body power as well as the ability to recruit motor units. It is performed from the half squat position with a knee angle of $90^{\circ}$; after a brief pause, the subject performing the test jumps upward as high as possible. The CMJ is a test used to assess explosive strength with reutilization of elastic energy and takes advantage of the myotatic reflex (7). The test starts with a preparatory movement of knee extension going down to a $90^{\circ}$ knee flexion and, without pausing, jumping upward as high as possible. Both jumps were performed without use of the arms; subjects were asked to keep their hands on their hips. Elevation of the center of gravity (height in meters) above ground level was calculated for both tests as flight time $\left(t_{v}\right)$ in seconds, applying the laws of ballistics:

$$
\mathrm{H}=\mathrm{t}_{\mathrm{v}}^{2} \cdot \mathrm{g} \cdot 8^{-1}(\mathrm{~m}) \text {; }
$$

where " $\mathrm{h}$ " is height, and " $\mathrm{g}$ " is gravitational acceleration $\left(9.81 \mathrm{~m} \cdot \mathrm{s}^{-2}\right)$ 


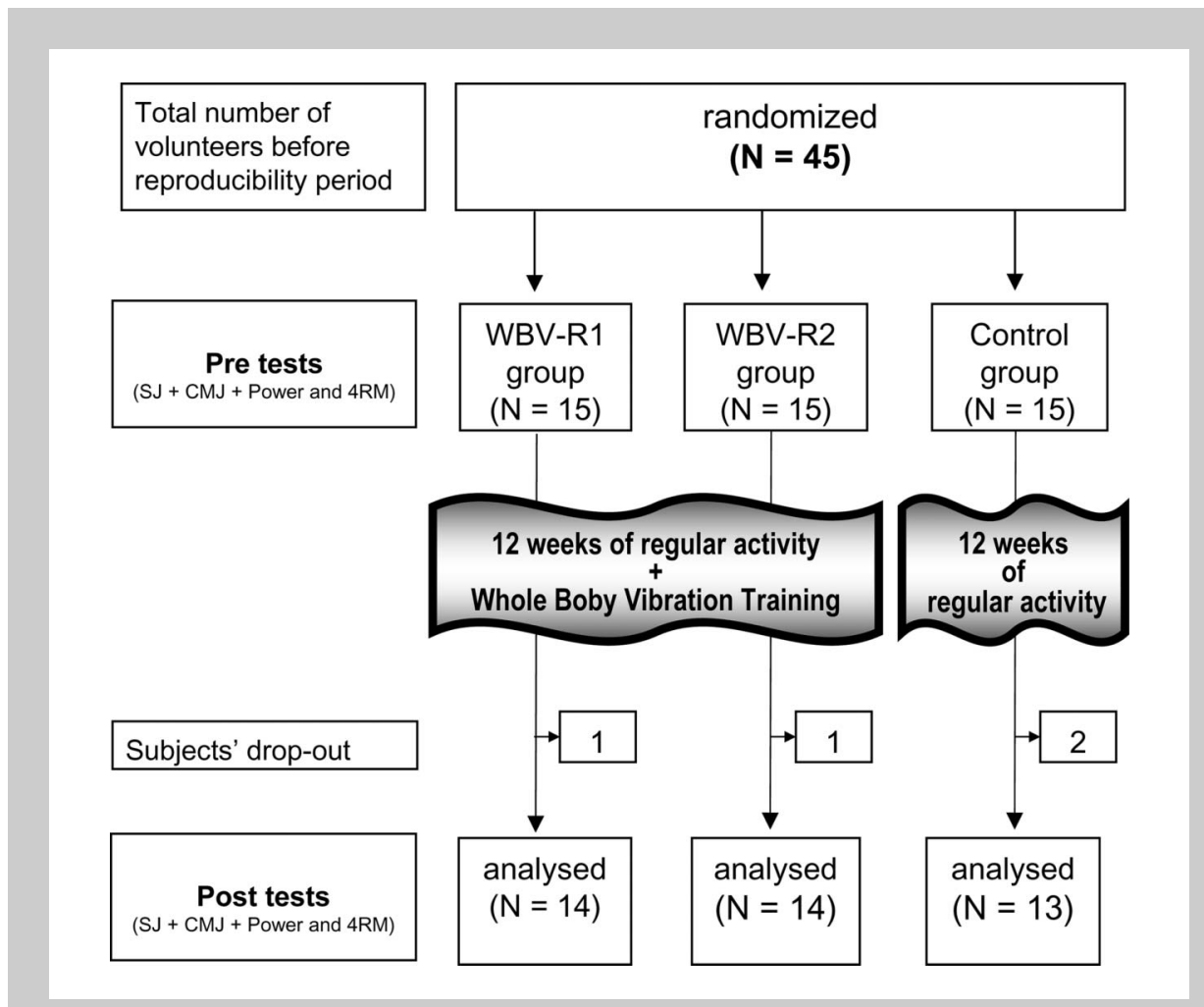

Figure 4. Data-recording protocol for Study II.
The jump flight time was measured on a digital chronometer connected to the platform of the the MuscleLab system.

Three jumps were performed for each type, the best result being used for statistical analysis.

Muscle Power. Although subjects had previously performed the jump tests, they performed a set of 8 repetitions at loads of $30-40 \%$ of the perceived maximum as a specific warm-up.

Lower-body maximal power was assessed using the $\mathrm{Mu}-$ scleLab system. Subjects were placed in a half squat position, with shoulders touching the bar; the starting knee angle for movement execution was set at $90^{\circ}$. When told to do so, subjects performed a concentric extension of the leg muscles (extensors of hip, knee, and ankle) starting from the flexed knee position and reaching full extension at $180^{\circ}$; this movement was performed

TABLE 2. Means and SD for performance test parameters: 10 minutes before (Pre) and 5 minutes after (Post) whole body vibration with 3 different recovery times: 1,2 , and 3 minutes, with respective pvalues; confidence interval $=95 \%$.

\begin{tabular}{|c|c|c|c|c|c|}
\hline \multirow[b]{2}{*}{ Minutes } & \multicolumn{2}{|c|}{ Mean $(S D)$} & \multirow{2}{*}{$\begin{array}{c}\text { Significance } \\
p^{\dagger}\end{array}$} & \multicolumn{2}{|c|}{$\begin{array}{l}\text { 95\% confidence } \\
\text { interval }\end{array}$} \\
\hline & Pre $^{*}$ & Post & & Lower & Upper \\
\hline \multicolumn{6}{|c|}{ Squat jump (cm) } \\
\hline 1 & $36.37(3.25)$ & $37.11(3.85)$ & 0.018 & 0.13 & 1.33 \\
\hline 2 & 36.57 (3.74) & 38.39 (3.59) & 0.001 & 1.34 & 2.28 \\
\hline 3 & $36.80(4.09)$ & $36.99(4.21)$ & 0.275 & -0.15 & 0.52 \\
\hline \multicolumn{6}{|c|}{ Countermovement jump (cm) } \\
\hline 1 & $40.02(4.11)$ & 40.91 (4.09) & 0.033 & 0.07 & 1.66 \\
\hline 2 & $39.81(4.67)$ & $42.10(4.97)$ & 0.001 & 1.61 & 2.95 \\
\hline 3 & $40.12(4.48)$ & 40.24 (4.63) & 0.491 & -0.23 & 0.47 \\
\hline \multicolumn{6}{|c|}{ Power (W) } \\
\hline 1 & $1,300.95(191.23)$ & 1,329.69 (190.67) & 0.028 & 3.28 & 54.18 \\
\hline 2 & $1,288.60(189.26)$ & $1,347.01(184.26)$ & 0.001 & 31.37 & 85.44 \\
\hline 3 & 1,295.97 (182.02) & $1,298.22(191.81)$ & 0.863 & -24.18 & 28.68 \\
\hline
\end{tabular}

*No differences between pretest values.

$\dagger$ Analysis of variance with repeated measures. 

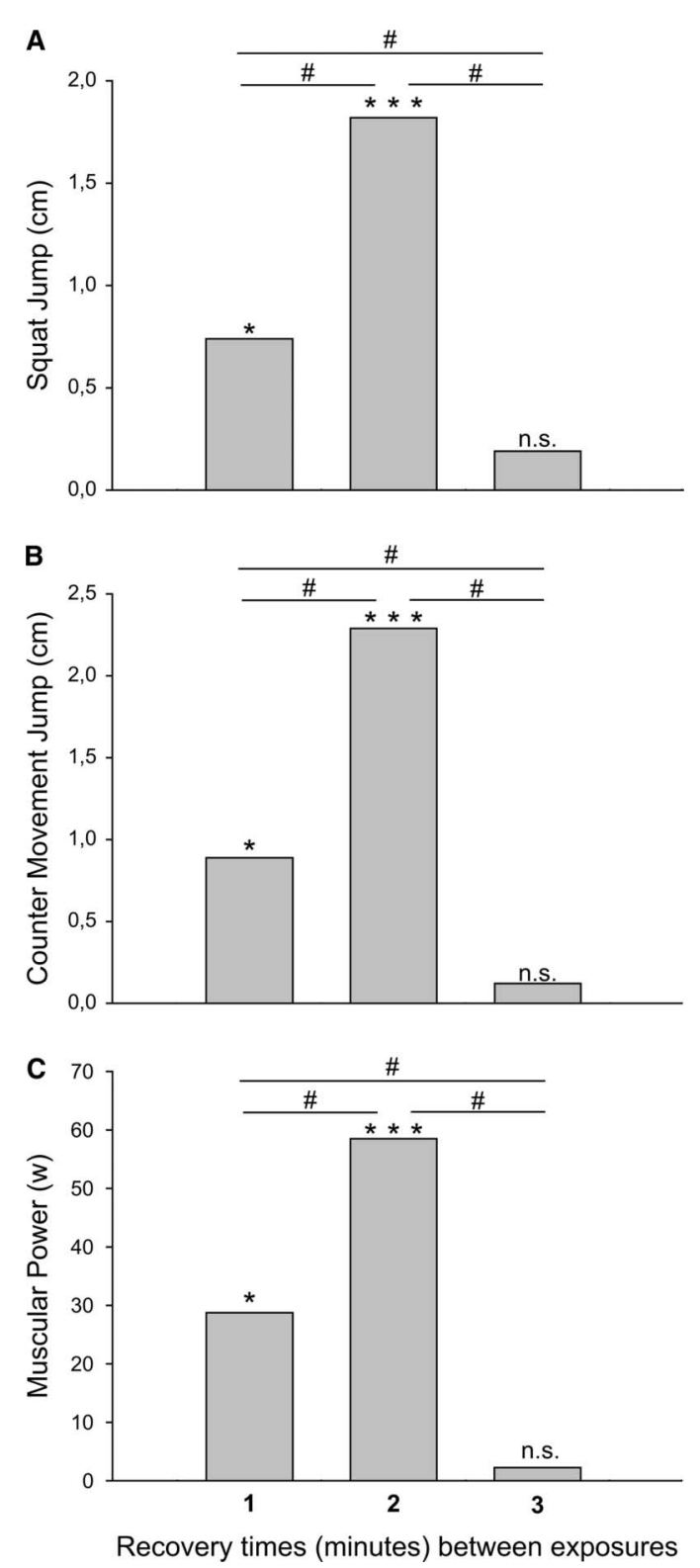

Figure 5. Mean difference between post-test and pretest results in Study I (acute): A) squat jump, B) countermovement jump, and C) muscular power, using 3 different recovery times between exposures to whole body vibration. *Mean differences significant for $p \leq 0.05$. ${ }^{* *}$ Mean differences significant for $p \leq 0.01$. \#Mean intergroup difference significant for $p \leq 0.05$.

against a resistance determined by weight plates added to both ends of the bar. Subjects were instructed to perform a purely concentric action from the starting point, keeping shoulders at an abducted position of $90^{\circ}$ to assure consistency of shoulder and elbow joints during execution of the movement (42).

Subjects were also asked to keep the trunk as erect as possible throughout the movement. Because this test was used for estimating maximal power, subjects were asked to perform the movement as quickly as possible (53).

All tests were performed using a Multipower (GervaSport, Madrid, Spain) apparatus, designed for performance of squat exercises in which the bar is displaced only in the vertical position as allowed by linear bearings. Four different loads added to body weight were used for estimating both maximal and mean power: $25,45,65$, and $85 \mathrm{~kg}$. Three trials were performed for each load, and the best result (maximum average speed) was used for subsequent analysis. During the test, data were collected regarding bar displacement, maximum average speed $(\mathrm{m} / \mathrm{s})$, and average power (watts), using the lineal encoder built into the MuscleLab system, whose internal microprocessor works at a resolution of $10 \mu \mathrm{s}$. As the load is moved, the optical transducer signal interrupts the microprocessor at every $0.07 \mathrm{~mm}$ displacement. Power calculations were performed as previously described (2). Average power was calculated by means of the range of motion used to perform a whole repetition.

4RM. Load capabilities were calculated for $4 \mathrm{RM}$ in the half-squat exercise using a Multipower (GervaSport, Madrid, Spain) machine equipped with calibrated disks and the standard protocol for submaximal strength testing developed by Kraemer and Fry (33). The cadence used was 3 seconds per exercise $(1.5 \mathrm{~s}$ for the eccentric phase and $1.5 \mathrm{~s}$ for the concentric phase) controlled by a digital metronome (MA-30, Korg, Tokyo, Japan).

\section{Study Design and Procedure}

Vibration parameters for Study I and Study II were as follows: 6 exposures of 60 seconds each, frequency $30 \mathrm{~Hz}$, shown to be most effective $(12,18)$, amplitude (peak-to-peak displacement) $4 \mathrm{~mm}$. Recovery time was the only variable in both studies. Vibration was applied using a vibrating platform producing sinusoidal oscillations (Nemes, Ergotest, Rome, Italy) (Figure 2). Subjects adopted a squatting position, knees flexed at $100^{\circ}$, as measured by a manual goniometer. To avoid bruising, all subjects wore trainers for vibration exercises. To avoid variations in vibration transmission, subjects were asked to wear the same footwear at all training sessions.

Study I. Recovery times between exposures varied during each session and were randomly assigned. All subjects were exposed to the 3 different recovery times: 1, 2, and 3 minutes. The rest period between sessions was a minimum of 72 hours to avoid carryover effects from previous sessions. Vibration was applied after the warm-up and tests described earlier; postvibration tests were also performed (Figure 3 ).

Study II. In this longitudinal study, all subjects were subjected to the same prestudy and poststudy tests. Pretest data were recorded 3 days before the start of training and poststudy data 3 days after the last training session to avoid the acute effects of WBV. All subjects completing the study attended all 12 scheduled training sessions (100\%). During the study, groups 
TABLE 3. Means and SD for performance test parameters before (Pre) and after (Post), 4 weeks' whole body vibration training using different recovery times: 1 minute (WBV-R1) and 2 minutes (WBV-R2), with respective $p$ values, confidence interval ( $\mathrm{Cl} 95 \%)$, and effect size.

\begin{tabular}{|c|c|c|c|c|c|c|}
\hline \multirow[b]{2}{*}{ Group } & \multicolumn{2}{|c|}{ Mean $(S D)$} & \multirow{2}{*}{$\begin{array}{c}\text { Significance } \\
p^{\dagger}\end{array}$} & \multicolumn{2}{|c|}{$\begin{array}{l}95 \% \text { confidence } \\
\text { interval }\end{array}$} & \multirow[b]{2}{*}{ Effect size } \\
\hline & Pre $^{\star}$ & Post & & Lower & Upper & \\
\hline \multicolumn{7}{|c|}{ Squat jump (cm) } \\
\hline WBV-R1 & $35.37( \pm 3.09)$ & $38.61( \pm 2.94)$ & 0.001 & 2.16 & 4.30 & 1.04 \\
\hline WBV-R2 & $36.49( \pm 3.98)$ & $38.08( \pm 4.46)$ & 0.008 & 0.44 & 2.73 & 0.40 \\
\hline C & $35.28( \pm 3.86)$ & $35.57( \pm 4.91)$ & 0.866 & -1.09 & 1.29 & 0.07 \\
\hline \multicolumn{7}{|c|}{ Countermovement jump (cm) } \\
\hline WBV-R1 & $39.70( \pm 3.45)$ & $42.64( \pm 4.17)$ & 0.001 & 2.02 & 3.85 & 0.85 \\
\hline WBV-R2 & $39.68( \pm 5.15)$ & $41.33( \pm 5.17)$ & 0.002 & 0.66 & 2.63 & 0.32 \\
\hline C & $39.31( \pm 4.77)$ & $39.68( \pm 4.98)$ & 0.170 & -0.31 & 1.73 & 0.07 \\
\hline \multicolumn{7}{|c|}{4 repetition maximum $(\mathrm{kg})$} \\
\hline WBV-R1 & $155.32( \pm 22.70)$ & $175.92( \pm 25.92)$ & 0.001 & 11.02 & 26.19 & 0.90 \\
\hline WBV-R2 & $157.25( \pm 23.31)$ & $174.27( \pm 24.48)$ & 0.021 & 2.229 & 30.11 & 0.53 \\
\hline \multicolumn{6}{|c|}{ Muscular power (W) } & 0.04 \\
\hline WBV-R1 & $1,293.80( \pm 140.32)$ & $1,390.74( \pm 168.51)$ & 0.003 & 35.99 & 157.88 & 0.69 \\
\hline WBV-R2 & $1,292.20( \pm 228.85)$ & $1,358.24( \pm 234.35)$ & 0.048 & 0.56 & 131.49 & 0.28 \\
\hline C & $1,268.24( \pm 241.38)$ & $1,282.28( \pm 288.12)$ & 0.338 & 48.77 & 130.31 & 0.04 \\
\hline
\end{tabular}

${ }^{\star}$ No difference between pretest values.

$\dagger$ Analysis of variance $(3 \times 2)$.

WBV with 1-minute recovery periods between exposures (WBV-R1) and WBV with 2-minute recovery periods between exposures (WBV-R2), underwent vibration training, whereas subjects in the control group continued with their usual sporting activities, controlled by a self-recording questionnaire (Figure 4).

Vibration training sessions took place 3 days a week (Monday, Wednesday, and Friday) for 4 weeks. After a standing stretching program for femoral quadriceps, hamstrings, and triceps surae, subjects started the vibration training session. The 2 groups undergoing vibration training, WBV-R1 and WBV-R2, rested for 1 and 2 minutes, respectively, between $\mathrm{WBV}$ exposures.

\section{Reproducibility of Variables}

Tests were repeated over 3 different days (Monday, Wednesday, and Friday) in the week before training. The intraclass correlation values (interday) were $\mathrm{SJ}=0.93, \mathrm{CMJ}=$ $0.96,4 \mathrm{RM}=0.94$, and power $=0.95$.

\section{Statistical Analyses}

Traditional statistical methods were used to calculate means and SD. Sample normality was calculated using the ShapiroWilks test. An analysis of variance and the Bonferroni adjustment for multiple comparisons were used to compare mean values. The following symbols were used to denote statistical significance: $p \leq 0.05\left(^{*}\right), p \leq 0.01\left(^{* *}\right), p \leq 0.001$ $\left(^{* *}\right)$. The significance level was set at $p \leq 0.05\left(^{*}\right)$; the SPSS
10.0 package for Windows (Chicago, IL, USA) was used for all statistical tests.

\section{Results}

Results of the acute and chronic studies are presented separately for a clearer understanding.

\section{Study I}

The values obtained for the 3 parameters tested (SJ, CMJ, and power) are shown in Table 2. No differences were recorded in pretest values for any of the randomly applied recovery times.

SJ, CMJ, and Power. The 3 tests yielded similar results: significant increases were observed for the application of both 1 - $(p<0.05)$ and 2 -minute $(p<0.01)$ recovery periods, whereas no difference was noted for application of the 3 -minute recovery period. Post hoc analysis displayed significant differences between all groups for poststudy versus prestudy increases (Table 2) (Figure 5).

\section{Study II}

Values obtained for the 4 parameters tested (SJ, CMJ, 4RM, and power) after 4 weeks' training are shown in Table 3 . As in Study I, there were no differences in prestudy values among the 3 groups (WBV-R1, WBV-R2, and control), to which subjects had been randomly assigned.

SJ, CMJ, and Power. A similar pattern was observed in the results obtained for the 3 tests in the WBV-R1 and WBV-R2 

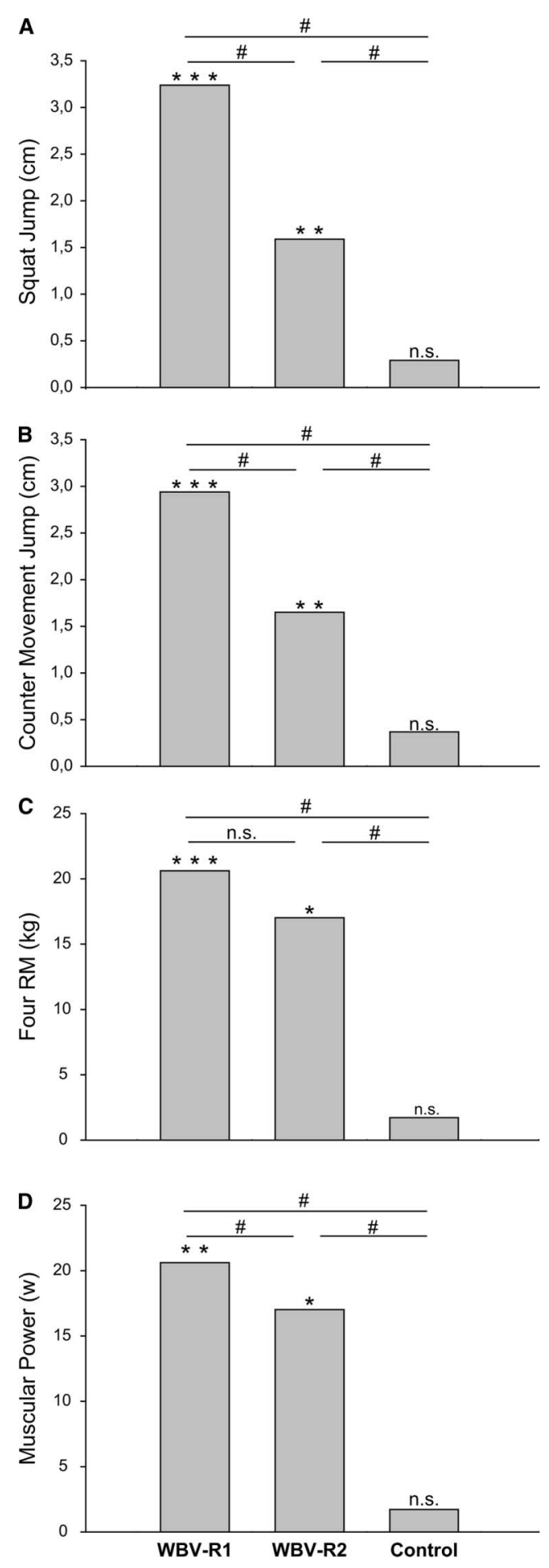

Figure 6. Mean between post-test and pretest results in Study II (cumulative): A) squat jump, B) countermovement jump, C) 4 repetition maximum, and D) muscular power for different recovery times during exposure to whole body vibration; 1 minute (WBV-R1), 2 minutes (WBVR2) y control. *Mean differences significant for $p \leq 0.05$. ${ }^{* *}$ Mean differences significant for $p \leq 0.01$. \#Mean intergroup difference significant for $p \leq 0.05$.

groups; the control group displayed no significant differences. Both WBV-R1 and WBV-R2 showed statistically significant increases for $\mathrm{SJ}, \mathrm{CMJ}$, and $\mathrm{P}$; when comparing both groups, WBV-R1 showed significant differences with regard to WBV-R2 $(p<0.05)$ in all analyzed parameters. Post hoc analysis revealed significant differences between all groups for poststudy versus prestudy increases (Table 3) (Figure 6).

$4 R M$. Muscle strength measured by means of the $4 \mathrm{RM}$ test showed significant difference for WBV-R1 $(p<0.01)$ and for WBV-R2 $(p<0.05)$ between prestudy and poststudy tests; no differences were found for the control group. Post hoc analysis showed significant differences between the control group and both experimental groups but not between the latter (Table 3) (Figure 6).

\section{Discussion}

The main finding was that short recovery times between exposures to WBV enhanced the acute response of muscle performance to WBV. Moreover, the cumulative effect of WBV training reduced the effective recovery time.

All repeated or intermittent exercise programs intended to develop either strength or endurance should take into account 4 factors: volume, duration, intensity, and recovery (57). A number of articles have analyzed and suggested optimum recovery times after strength training exercises $(62,57,23,46,45,63)$, as well as investigating their effect on perceived exertion (64). However, this appears to be the first study designed to assess the effects of different recovery times after WBV training. Recovery, which varies depending on the duration and intensity of the stimulus, is aimed at restoring the muscle to its initial prestimulus state (22). Therefore, an adequate recovery is essential to ensure that the muscle eliminates activation-induced fatigue and returns to a state favoring the successful undertaking of a new activity (27). On the other hand, an incomplete recovery hinders the muscle's ability to meet the demands of further contraction (55). It has to be noted, however, that the recovery process is not linear over time; at certain moments in the process there is a transient increase in muscle contractile performance with respect to the levels that would be achieved from a state of total rest. This phenomenon is termed PAP or moment of muscle supercompensation $(59,1,55,57)$. Based on this, Study I mainly aimed to achieve this state in the absence of muscle fatigue by identifying the optimum recovery time after stimulation of leg extensor muscles by WBV training.

The results suggest that a 2-minute recovery period between exposures is the most effective in prompting acute enhancement of jump ability and muscle power. A 1-minute recovery period prompted significant changes for both parameters $(p<0.05)$, a finding also noted by other authors both for SJ (13) and for $\mathrm{CMJ}(5,47)$. Increases in muscle power, similar to the ones observed in the present study, have also been reported by Bosco et al. $(4,5)$ previously. The jump and power results obtained here suggest that a 2-minute 
recovery period leads to greater optimization of the training session; in fact, improvements were significantly greater $(p<0.05)$ than after 1 minute. By contrast, Ruiter et al. (52), using a WBV protocol including a 2 -minute recovery period, recorded a decline in jump ability (CMJ). This may be because they used a vibration amplitude twice that used here (8 $\mathrm{mm}$ vs. $4 \mathrm{~mm})$. Cardinale et al. (11) have shown that amplitude has a decisive influence on the response to WBV training. Therefore, the amplitude used by Ruiter et al. may have prompted a greater muscle fatigue, thus requiring a longer recovery period to achieve peak PAP.

Strikingly, although 1-minute and 2-minute recovery periods appeared to be effective, the 3-minute recovery interval led to no significant improvements. For the subjects tested here, the longer recovery interval would appear to be excessive for the stimulus used, prompting the loss of the improvement achieved after a shorter interval. Cormie et al. (15) have suggested that WBV could be used for warm-up purposes; because short recovery times $(1,2 \mathrm{~min})$ ensured in our study an enhanced PAP, we could postulate that they could be used when WBV is used as part of a warm-up routine.

WBV stimulus entails subjecting the body to small oscillations that prompt small changes in the length of skeletal muscle fibers; these, in turn, activate reflex mechanisms. This gives rise to activation of Ia afferent fibers through spindle excitation, an effect similar to that prompted in the tonic vibration reflex (TVR) described by Hagbarth and Eklund (24), and to improved Ia loop activity (8). Other authors have pointed to the possible existence of an excitatory inflow over short connections between muscle spindles and motorneurons (34) and to increased activation of motor areas of the central nervous system (39). Because of these complex associations, WBV training provides intense stimulus of the skeletal muscle, even though the exact mechanism is still not entirely understood.

Training, however, rarely pursues such short-term goals, seeking rather to achieve a cumulative adaptive response (Study II) as a means of stabilizing useful muscle behavior during sporting activity. This is known as sequential adaptation or chronic adaptation (57).

Most studies addressing the adaptive response to strength training can be classed under 2 main headings: those focusing on structural changes and those dealing with alterations taking place in the central or peripheral nervous system (32). Chronologically speaking, it is widely accepted that this adaptive response starts with changes in the nervous system and ends with morphologic changes involving a number of structures (mainly muscles, bones, and tendons). This is clear from reports that short-term strength training increases muscle strength without apparently modifying muscle structures (40,54). Any potentially strength-enhancing changes taking place during short-term training are mainly nerve related. For that reason, no morphologic or anthropometric variables were monitored in the present study with a view to charting muscle hypertrophy in activated areas.
A key feature of the present study is that the subjects, although routinely engaged in a range of sporting activities, had no prior experience of weight training. For that reason, body weight was used for loading over the 4-week training period. Even so, WBV prompted a significant improvement in jump ability, strength, and power. Some studies of conventional short-term strength training report that, over such short periods, no significant improvement was observed in jump ability either in subjects similar to those used here (25) or in top-level volleyball players (6).

However, in the present study, WBV appears to improve such characteristics after a short-term treatment, which appears to agree with the theory that WBV technology has beneficial effects in a wide range of sporting activities (10), as well as in motor rehabilitation (9) and general health (14). Moreover, the 2 protocols tested in Study II (WBV-R1 and WBV-R2) prompted significant improvements in jump ability, the best results being achieved with the shortest recovery period ( $1 \mathrm{~min}$ ). This would appear to contradict the finding reported for the acute study (Study I) in which the best results for jump ability were recorded using a 2-minute recovery period. However, if acute muscle response had been charted over the whole 4-week period, the recovery period providing the best acute response, initially 2 minutes, might gradually have diminished as the number of training sessions increased because of cumulative adaptation. This hypothesis would be supported by the results obtained by Bosco et al. (3), which reflect an improvement of jump ability in athletes, similar to the ones obtained in the present study, after 10 sessions in 2 weeks of 90 -second-exposure and 40 -secondrecovery periods.

Although most studies $(29,4,12,13,52,47)$, including the present one, have evaluated the effect of WBV on standing subjects, there have also been reports of improvements of jump ability in subjects undergoing dynamic exercises. Indeed, Ronnestad (50) reported a significant increase in CMJ ability after 5 weeks of training using loads of up to $90 \%$ of $1 \mathrm{RM}$ in the group subjected to WBV.

As with jump ability, the present study recorded significant increases in muscle power, particularly after a 1-minute recovery period. Although a number of articles have addressed the acute effect of WBV training on muscle power $(4,30)$, there has been very little specific research into the cumulative effect. Likewise, muscle strength (4RM) was also enhanced by WBV training. Other authors report positive effects of WBV on muscle strength over a relatively short period $(29,58)$. In line with our results, Mester et al. (38), comparing conventional strength training using 2 dynamicexercise protocols (half squat with $50 \%$ of $1 \mathrm{RM}$ ) with WBV ( 2 and $4 \mathrm{~mm}$ ), reported significantly better results both in maximum isometric force and in muscle strength for the groups using WBV (particularly at an amplitude of $4 \mathrm{~mm}$ ). Both training protocols (WBV-R1 and WBV-R2) showed significant increases in muscle strength $(13.26 \%, p<0.001$; $10.82 \%, p<0.05$, respectively). Although not statistically 
Journal of Strength and Conditioning Research" $\mid$ www.nsca-jscr.org

significant, there were differences observed in the strength increase magnitude the 2 groups experienced (effect size $=$ 0.90 vs. 0.53 , respectively).

It has been suggested that increased muscle strength may be caused largely by a neuromuscular activation linked to the TVR (10), although a number of articles point to other possible explanations. Issurin (28) reports that the cumulative effect of WBV stimulus improves monosynaptic stretch reflexes induced by afferent signals from muscle spindles, as well as reducing the inhibiting impact of Golgi tendon organs located at myotendinous junctions. Other possible causes may include a change in perceived exertion (35), improved motorneuron excitability $(10,20,49)$, increased muscle temperature and increased blood flow $(44,30)$, possible improvements in the anabolic hormone balance $(37,10)$, and muscle hypertrophy $(41,21)$.

Necking et al. (41) found that the cumulative effect of WBV increased the size of both slow-twitch and fast-twitch fibers in rats. It is not clear why the training protocol including a 1-minute recovery period proved more effective in Study II. Further research is undoubtedly required into training-load design techniques when using vibratory platforms. The literature on the effects of WBV training still contains disparate results, largely because of the use of different amplitudes, frequencies, exposure times, total number of exposures, and recovery intervals. The parameters selected for use here, with the exception of amplitude, had been proved suitable in previous studies by our research group $(16,17)$.

To summarize, WBV training appears to be a safe and effective method of achieving a favorable acute response in muscle performance. In moderately active subjects, a 2minute recovery period appears most effective in maximizing PAP and thus improving acute response. Optimum recovery times, however, may be modified as a result of systematic WBV training. Twelve WBV training sessions reduced optimum recovery times to 1 minute for jump and power tests. Both recovery times ( $1 \mathrm{~min}$ and $2 \mathrm{~min}$ ) appeared equally effective in the case of $4 \mathrm{RM}$.

\section{Practical Applications}

It is considered that WBV training sessions for moderately active subjects should include 2 different recovery times after exposure: a recovery time of roughly 1 minute to improve short-term performance in actions requiring high explosive force or high levels of maximum dynamic strength, thus optimizing the limited time generally available for training and a longer recovery period (approximately $2 \mathrm{~min}$ ) when $\mathrm{WBV}$ is used to enhance muscle activation during warm-up.

\section{REFERENCES}

1. Abbate, F, Sargeant, AJ, Verdijk, PW, and De Haan, A. Effects of high-frequency initial pulses and posttetanic potentiation on power output of skeletal muscle. J Appl Physiol 88: 35-40, 2000.

2. Bosco, C, Belli, A, Astrua, M, Tihanyi, J, Pozzo, R, Kellis, S, Tsarpela, O, Foti, C, Manno, R, and Tranquilli, C. A dynamometer for evaluation of dynamic muscle work. Eur J Appl Physiol 70: 379-386, 1995.

3. Bosco, C, Cardinale, M, Tsarpela, O, Colli, R, Tihanyi, J, Von Duvillard, SP, and Viru, A. The influence of whole body vibration on jumping performance. Biol Sport 15: 157-164, 1998.

4. Bosco, C, Colli, R, Introini, E. Cardinale, M, Iacovelli, M, Tihanyi, J, Von Duvillard, SP, and Viru, A.SP, and Viru, A. Adaptive responses of human skeletal muscle to vibration exposure. Clin Physiol 16: 317322, 1999 .

5. Bosco, C, Iacovelli, M, Tsarpela, O, Cardinale, M, Bonifazi, M, Tihanyi, J, Viru, M, De lorenzo, A, and Viru, A. Hormonal responses to whole-body vibration in men. Eur J Appl Physiol 81: 449-454, 2000.

6. Bosco, C, Komi, PV, and Ito, A. Prestretch potentiation of human skeletal muscle during ballistic movement. Acta Physiol Scand 111: 135-140, 1981.

7. Bosco, C, Luhtanen, P, and Komi, PV. A simple method for measurement of mechanical power in jumping. Eur J Appl Physiol 50: 273-282, 1983.

8. Burke, D, Hogbarth, E, Lofstedt, L, and Wallin, BG. The response of human muscle spindle endings to vibration during isometric contractions. J Physiol 261: 695-711, 1976.

9. Cafarelli, E, Sim, J, Carolan, B, and Liebesman, J. Vibratory massage and short-term recovery from muscular fatigue. Int J Sports Med 11: 474-478, 1990.

10. Cardinale, $\mathrm{M}$ and Bosco, $\mathrm{C}$. The use of vibration as an exercise intervention. Exerc Sport Sci Rev 31: 3-7, 2003.

11. Cardinale, M, Leiper, J, Erskine, J, Milroy, M, and Bell, S. The acute effects of different whole body vibration amplitudes on the endocrine system of young healthy men: a preliminary study. Clin Physiol Funct Imaging 26: 380-384, 2006.

12. Cardinale, M and Lim, J. Electromyography activity of vastus lateralis muscle during whole-body vibrations of different frequencies. J Strength Cond Res 17: 621-624, 2003.

13. Cardinale, $\mathrm{M}$ and Lim, J. The acute effects of two different whole body vibration frequencies on vertical jump performance. Med Sport 56: 287-292, 2003.

14. Cardinale, $M$ and Rittweger, J. Vibration exercise makes your muscles and bones stronger: fact or fiction? J Br Menopause Soc 12: 12-18, 2006.

15. Cormie, P, Deane, RS, Triplett, NT, and Mcbride, JM. Acute effects of whole-body vibration on muscle activity, strength, and power. $J$ Strength Cond Res 20: 257-261, 2006.

16. Da Silva, M, Benno Becker, JR, García-Manso, JM, and Lancho, JL. Effect of different training of whole body vibration on muscular performance. Fiet Bulliten 77: 179, 2007.

17. Da Silva, M, Benno Becker, JR, García-Manso, JM, and Lancho, JL. Effect of different exposure times to whole body vibration on muscular performance. Fiet Bulliten 77: 180, 2007.

18. Da Silva, M, Núñez, VM, Vaamonde, DM, Fernández, JM, Poblador, MS, Lancho, JL. Effects of different frequencies of whole body vibration on muscular performance. Biol Sport 23: 267-282. 2006.

19. Da Silva, M, Vaamonde, DM, and Padullés, JM. [Effects of mechanical vibration training on neuromuscular performance]. APUNTS Educació Física Esports 84: 39-47, 2006.

20. Delecluse, C, Roelants, M, and Verschueren, S. Strength increase alters whole body vibration compared with resistance training. Med Sci Sport Exerc 35: 1033-1041, 2003.

21. Falempin, $M$ and In-Albon, SF. Influence of brief daily tendon vibration on rat soleus muscle innon-weight-bearing situation. J Appl Physiol 87: 3-9, 1999.

22. Giacomoni, M, Billaut, F, and Falgairette, G. Effects of the time of day on repeated all-out cycle performance and short-term recovery patterns. Int J Sports Med 27: 468-474, 2006. 
23. Fleck, S and Kraemer, WJ. Designing Resistance Training Programs. Champaign, IL: Human Kinetics, 1997.

24. Hagbarth, KE and Eklund, G. Motor effects of vibratory stimuli in man. In: Proceedings of the First Nobel Symposium on Muscular Afferents and Motor Control. Granit, R, ed. Stockholm: Almqvist and Wiksell, 1965. pp. 177-186.

25. Hakkinen, $\mathrm{K}$ and Komi, PV. Effect of explosive type strength training on electromyography and force production characteristics of leg extensor muscle during concentric and various stretch-shorting exercise. Scand J Sports Sci 7: 65-76, 1985.

26. Hamada, T, Sale, DG, Macdougall, JD, and Tarnopolsky, MA. Postactivation potentiation, fiber type, and twitch contraction time in human knee extensor muscles. JAppl Physiol 88: 2131-2137, 2000.

27. Hodgson, M, Docherty, D, and Robbins, D. Post-activation potentiation: underlying physiology and implications for motor performance. Sports Med 35: 585-595, 2005.

28. Issurin, VB. Vibrations and their applications in sport. A review. J Sports Med Phys Fitness 45: 324-336, 2005

29. Issurin, VB, Liebermann, DG, and Tenenbaum, G. Effect of vibratory stimulation training on maximal force and flexibility. J Sports Sci 12 561-566, 1994.

30. Issurin, VB and Tenenbaum, G. Acute and residual effects of vibratory stimulation on explosive strength in elite and amateur athletes. J Sports Sci 17: 177-182, 1999.

31. Komi, P and Bosco, C. Utilization of stored elastic energy in leg extensor muscles by men and women. Med Sci Sport Exerc 13: 190192, 1978.

32. Kraemer, WJ, Fleck, SJ, and Evans, WJ. Strength and power training: physiological mechanisms of adaptation. Exerc Sports Sci Rev 24: 363-397, 1996.

33. Kraemer, WJ and Fry, AC. Strength testing: development and evaluation methodology. In: Physiological Assessment of Human Fitness. Maud, P and Foster, C, eds. Champaign, IL: Human Kinetics, 1995.

34. Lebedev, MA and Polyakov, AV. Analysis of surface EMG of human soleus muscle subjected to vibration. J Electromiogr Kinesiol 2: 1-10, 1991.

35. Lieberman, DG and Issurin, V. Effort perception during isotonic muscle contractions with superimposed mechanical vibratory stimulation. J Hum Move Stud 32: 171-186, 1997.

36. Luo, J, Mcnamara, B, and Moran, K. The use of vibration training to enhance muscle strength and power. Sports Med 35: 23-41, 2005.

37. McCall, GE, Grindeland, RE, Roy, RR, and Edgerton, VR. Muscle afferent activity modulates bioassayable growth hormone in human plasma. J Appl Physiol 89: 1137-1141, 2000.

38. Mester, J, Kleinoder, $\mathrm{H}$, and Yue, Z. Vibration training: benefits and risks. J Biomech 39: 1056-1065, 2006.

39. Milner-Brown, HS, Stein, RB, and Lee, RG. Synchronization of human motor units: possible roles of exercise and supraspinal reflexes. Electroencephalogr Clin Neurophys 8: 245-254, 1975.

40. Moritani, Tand De Vries, H. Neural factors versus hipertrophy in the time course of muscle strength gain. Am J Phys Med 58: 115-130, 1979.

41. Necking, LE, Lundstrom, R, Lundborg, G, Thornell, LE, and Friden, J. Skeletal muscle changes after short term vibration. Scand J Plast Reconstr Surg Hand Surg 30: 99-103, 1996.

42. Newton, RU, Murphy, AJ, Humphries, BJ, Wilson, GJ, Kraemer, WJ, and Häkkinen, K. Influence of load and stretching shortening cycle on the kinematics, kinetics and muscle activation that occurs during explosive upper-body movements. Eur J Appl Physiol 75: 333-342, 1997.

43. Nordlund MM and Thorstensson, A. Strength training effects of whole-body vibration? Scand J Med Sci Sports 17: 12-7, 2007.
44. Oliveri, DJ, Lynn, K, and Hong, CZ. Increased skin temperature after vibratory stimulation. Am J Phys Med Rehabil 68: 81-85, 1989.

45. Pincivero, DM and Campy, RM. The effects of rest interval length and training on quadriceps femoris muscle. Part I. Knee extensor torque and muscle fatigue. J Sports Med Phys Fitness 44: 111-118, 2004

46. Pincivero, DM, Lephart, SM, and Karunakara, RG. Effects of intrasession rest interval on strength recovery and reliability during high intensity exercise. J Strength Cond Res 12: 152-156, 1998.

47. Porta, J, Mas, J, Paredes, C, Izquierdo, E, Aliaga, J, and Martí, D. [Effects of one session of Whole Body Vibration on maximal and explosive strength in cyclists and junior jumpers]. Red 17: 4-14, 2003.

48. Rehn, B, Lidström, J, Skoglund, J, and Lindström, B. Effects on leg muscular performance from whole-body vibration exercise: a systematic review. Scand J Med Sci Sports 17: 2-11, 2007

49. Rittweger, J, Mutschelknauss, M, and Felsenberg, D. Acute changes in neuromuscular excitability after exhaustive whole body vibration exercise as compared to exhaustion by squatting exercise. Clin Physiol Funct Imaging 23: 81-86, 2003.

50. Ronnestad, BR. Comparing the performance-enhancing effects of squats on a vibration platform with conventional squats in recreationally resistance-trained men. $J$ Strength Cond Res 18 : 839-845, 2004.

51. Rooney, KJ, Herbert, RD, and Balnave, RJ. Fatigue contributes to the strength training stimulus. Med Sci Sports Exerc 26: 1160-1164, 1994.

52. Ruiter, CJ, Van der Linden, RM, Van der Zijden, MJ, Hollander, AP, and De Han, A. Short-term effects of Whole-body vibration on maximal voluntary isometric knee extensor force and rate of force size. Eur J Appl Physiol 88: 472-475, 2003.

53. Sahaly, R, Vandewalle, H, Driss, T, and Monod, H. Maximal voluntary force and rate of force development in humansimportance of instruction. Eur J Appl Physiol 85: 345-350, 2001.

54. Sale, DG. Neural adaptation to resistance training. Med Sci Sport Exerc 20: 135-145, 1988.

55. Sale, DG. Postactivation potentiation: role in human performance. Exerc Sport Sci Rev 30: 138-143, 2002.

56. Schlumberger, A, Salin, D, and Schmidtbleicher, D. [Strength training with superimposed vibrations]. Sportverletz Sportschaden 15 : 1-7, 2001.

57. Siff, M and Verkhoshansky, YV. Supertraining. Special Strength Training for Sporting Excellence. Escondido, CA: Sports Training, 1996.

58. Torvinen, S, Kannus, P, Sievanen, H, Jarvinen, TA, Pasanen, M, Kontulainen, S, Jarvinen, TL, Jarvinen, M, Oja, P, and Vuori, I. Effect of four-month vertical whole body vibration on performance and balance. Med Sci Sports Exerc 34: 1523-1528, 2002.

59. Vandervoort, AA, Quinlan, J, and Mccomas, AJ. Twitch potentiation after voluntary contraction. Exp Neurol 81: 141-152, 1983.

60. Verschueren, S, Roelants, M, Delecluse, C, Swinnen, S, Vanderschueren, D, and Boonen, S. Effect of 6-month whole body vibration training on hip density, muscle strength, and postural control in postmenopausal women: a randomized controlled pilot study. J Bone Miner Res 19: 352-359, 2004.

61. Viru, A. Adaptation in Sports Training. London, UK: CRL Press, 1995.

62. Weiss, LW. The obtuse nature of muscular strength: the contribution of rest to its development and expression. J Appl Sports Sci Res 5: 219-227, 1991.

63. Willardson, JM and Burkett, LN. A comparison of 3 different rest intervals on the exercise volume completed during a workout. J Strength Cond Res 19: 23-26, 2005.

64. Woods, S, Bridge, T, Nelson, D, Risse, K, and Pincivero, DM. The effects of rest interval length on ratings of perceived exertion during dynamic knee extension exercise. $J$ Strength Cond Res 18: 540-545, 2004 